CLINICAL STUDY

\title{
Fatal and non-fatal adverse events of glucocorticoid therapy for Graves' orbitopathy: a questionnaire survey among members of the European Thyroid Association
}

\author{
Claudio Marcocci, Torquil Watt ${ }^{1}$, Maria Antonietta Altea, Ase Krogh Rasmussen ${ }^{1}$, Ulla Feldt-Rasmussen ${ }^{1}$, \\ Jacques Orgiazzi ${ }^{2}$, Luigi Bartalena ${ }^{3}$ and for the European Group of Graves' Orbitopathy (EUGOGO) \\ Department of Endocrinology and Metabolism, University of Pisa, Via Paradisa 2, 56124 Pisa, Italy, ${ }^{1}$ Department of Medical Endocrinology, Copenhagen \\ University Hospital Rigshospitalet, Copenhagen, Denmark, ${ }^{2}$ Department of Endocrinology and Metabolism, Hopital Lyon Sud, and Claude Bernard \\ University, Lyon, France, and ${ }^{3}$ Department of Clinical Medicine, University of Insubria, Varese, Italy \\ (Correspondence should be addressed to C Marcocci; Email: claudio.marcocci@med.unipi.it)
}

\begin{abstract}
Objective: The objective of this study was to investigate the side effects of glucocorticoid (GC) therapy observed by European thyroidologists during the treatment of Graves' orbitopathy (GO).

Design: A questionnaire-based survey among members of the European Thyroid Association (ETA) who treat GO.

Results: A response was obtained from 128 ETA members of which 115 used GC therapy for GO. The majority of respondents $(83 / 115,72 \%)$ used intravenous (i.v.) GC, with a relatively wide variety of therapeutic regimens. The cumulative dose of methylprednisolone ranged between 0.5 and $12 \mathrm{~g}$ (median $4.5 \mathrm{~g}$ ) for i.v.GC and between 1.0 and $4.9 \mathrm{~g}$ (median $2.4 \mathrm{~g}$ ) for oral GC. Adverse events were often reported during oral GCs $(26 / 32,81 \%)$; most side effects were non-severe, but ten respondents reported severe adverse events (hepatic, cardiovascular, and cerebrovascular complications), including two fatal cases, both receiving a total of $2.3 \mathrm{~g}$ prednisone. Adverse events were less common in i.v.GC (32/83 respondents, 39\%), but mostly consisted of severe events, including seven fatal cases. All but one fatal event occurred in cumulative i.v.GC doses $(>8 \mathrm{~g})$ higher than those currently recommended. Conclusions: GCs are preferentially administered i.v. for the treatment of GO in Europe. Both oral and i.v.GC may be associated with severe adverse effects, including fatal cases, which are more frequently reported in daily or alternate day i.v.GC. IvGC therapy should be undertaken in centers with appropriate expertise. Patients should be carefully examined for risk factors before treatment and monitored for side effects, which may be asymptomatic, both during and after treatment.
\end{abstract}

European Journal of Endocrinology 166 247-253

\section{Introduction}

Glucocorticoids (GC), associated with or without orbital radiotherapy, represent the first-line treatment for moderate-to-severe and active Graves' orbitopathy (GO) (1). In clinical practice, it is still undefined whether clinicians in Europe utilize the i.v., oral, or local (subconjunctival or parabulbar) routes.

Randomized clinical trials $(2,3,4,5,6)$ and two recent meta-analyses $(7,8)$ have demonstrated a greater efficacy of the i.v. pulse treatment over oral GC. Indeed, in the last 10 years, the i.v. pulse route has gained wide acceptance, particularly in Europe, and is presently considered the treatment of choice for moderate-to-severe and active GO (1). Evidence-based demonstration of the optimal therapeutic regimen, however, is still lacking.

Although usually better tolerated than oral GC, i.v.GC treatment was associated with fatal acute liver failure and cardiovascular and cerebrovascular events $(9,10,11,12,13)$. In a workshop held at the 2007 Annual Meeting of European Thyroid Association (ETA) on this issue, more clinicians than expected reported severe complications of i.v.GC. For this reason, a group of members of the ETA and the European Group on Graves' Orbitopathy (EUGOGO) were assigned the task of further addressing this issue by developing a specific questionnaire and distributing it among the ETA members.

The aims of this questionnaire were to gather information on the choice of GC treatment in clinical practice for moderate-to-severe and active GO and, in particular, to evaluate its side effects. Members were instructed to report their actual practice, without considering the EUGOGO consensus statement on the management of GO that was published shortly before the survey was launched. 


\section{Materials and methods}

The format of the questionnaire was established by a group of experienced clinicians, all members of the ETA. It covered basic background information about the participating clinicians, including whether the respondents treated patients with GO, the approximate number of patients treated every year, the treatment of choice for moderate-to-severe and active GO, details about the treatment (e.g. dosing regimen, use of concomitant treatments, etc.), side effects of GC treatment, including fatalities, and information about the use of bone anti-resorptive therapy. Details concerning adverse events were collected initially by the questionnaire. Additional information about severe adverse events was then gathered by further e-mails.

The questionnaire was designed as an online survey with skip-patterns, using Esurveyspro.com (www.esurveyspro.com). The questionnaire consisted of 1-24 items, depending on the response pattern (e.g. respondents answering 'No' to the first item concerning whether he/she was treating patients with GO were not administered any additional items). After the initial survey development, a pretest was performed on five potential respondents and subsequent refinements were implemented. The full questionnaire is available upon request from the authors.

In June 2009, an invitation to participate in the survey containing a tagged link to the online survey was sent to all ordinary ETA members (i.e. those whose residence or place of work is in Europe or any country bordering the Mediterranean sea) whose e-mail address was available $(n=349)$. Two reminders were sent to non-responders.

On completion of the survey in October 2009, the response database was downloaded and analyzed using SAS 9.1 (14). Results were subsequently exported to Excel spreadsheets for reporting. Results are expressed as percent of answers, unless otherwise indicated.

\section{Results}

Two hundred and forty-five of the 349 invited ETA members $(70.2 \%)$ linked into the survey website, but only 148 gave a positive answer to the first question (Do you treat patients with GO in your clinic?). The remaining 97 members who linked into the website survey were not treating patients with GO (they were likely basic scientists). Of the 148 ETA members who replied positively to the first question, only 128 used GC in the treatment of patients with GO (subsequently termed 'treating clinicians'). Most responses came from Italy $(n=18)$ and Denmark $(n=14)$, followed by Germany $(n=9)$, Greece $(n=9)$, Turkey $(n=9)$, UK $(n=7)$, Sweden $(n=7)$, Bulgaria $(n=6)$, Poland $(n=6)$, and Serbia $(n=5)$.
Fifty of the treating clinicians $(39 \%)$ treated one to ten GO patients each year, 41 (32\%) treated 11-20 patients, and $37(29 \%)$ treated more than 20 patients. Of the 128 respondents treating patients with moderate-to-severe GO, 115 (90\%) used GC: 83 (72\%) used the i.v. route and $32(28 \%)$ the oral route and were the respective denominators for the following calculations. The remaining 13 respondents used other treatment modalities (e.g. orbital radiotherapy, etc.).

\section{Oral route}

Among the 32 ETA members who used oral GC, 29 (91\%) used a fixed initial dose and three (9\%) an initial dose based on body weight. The fixed initial dose was 20-40 mg prednisone (or prednisone equivalent) in seven, $40-75 \mathrm{mg}$ in 16 , and $>75 \mathrm{mg}$ in six cases. In most cases, the initial dose was continued for at least 2 weeks and then tapered off at 2-week intervals. The duration of treatment was longer than 3 months in most cases. The total dose ranged between 1 and $4.9 \mathrm{~g}$, median $2.4 \mathrm{~g}$.

Twenty-six out of the 32 respondents (81\%) using oral GC reported adverse events. Ten respondents reported severe adverse events (cardiovascular $(n=1)$, cerebrovascular $(n=3)$, and increase (greater than fourfold upper normal limit) in liver enzymes $(n=6)$ ) and 17 reported mild/moderate adverse events. One respondent reported both severe and mild-to-moderate adverse events. The latter included hyperglycemia, gain in body weight, depression, and cushingoid features. Detailed data were obtained from four patients with severe adverse events. Two patients died during oral GC therapy due to cerebrovascular events (Table 1). One of them was also treated for congestive heart failure. The total dose administered at the time of the fatal event was $2.3 \mathrm{~g}$ prednisone in both cases. One of the two patients with serious, but non-fatal, adverse events had pulmonary embolism and the other a greater than fourfold increase in liver enzymes.

\section{I.v. route}

The treatment schedules used by the 83 ETA members using i.v.GC are shown in Fig. 1. Sixty-eight $(82 \%)$ members used a fixed starting dose of equivalent methylprednisolone (ten members $(15 \%),<0.5 \mathrm{~g} ; 50$ members $(73 \%), 0.5-1 \mathrm{~g}$; and eight members $(12 \%)$ : $>1 \mathrm{~g})$. Treatment schedules were as follows: once weekly $(58 \%)$, twice weekly $(14 \%)$, three times weekly $(18 \%)$, twice a week on alternate weeks $(1 \%)$, or other schedule $(10 \%)$. Oral GC was administered either in the interpulse period by 16 or after the end of treatment in gradually tapered doses by 29 of these 83 ETA members. The cumulative dose of i.v.GC ranged between 0.5 and $12 \mathrm{~g}$, median $4.5 \mathrm{~g}$.

Thirty-two of the 83 ETA members using i.v.GC (39\%) reported adverse events. Of these 32 respondents, 


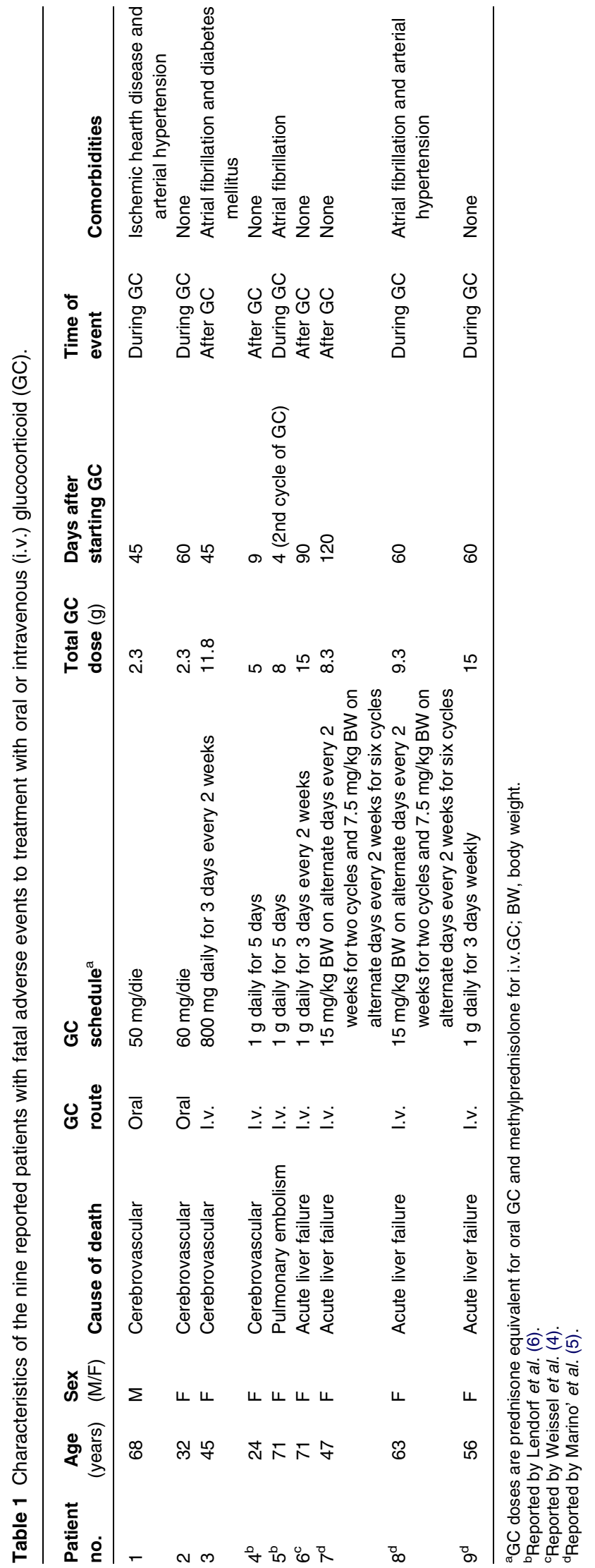

27 reported severe adverse events (cardiovascular $(n=10)$, cerebrovascular $(n=5)$, acute liver failure $(n=3)$, autoimmune encephalitis $(n=1)$ and increase (greater than fourfold upper normal limit) in liver enzymes $(n=8)$ ), and 14 reported mild-to-moderate adverse events. Nine respondents reported both mildto-moderate and severe adverse events. Detailed data were available for 32 patients with non-fatal severe adverse events (Table 2) and seven with fatal adverse events (Table 1). All fatal adverse events occurred in females. Three patients died during the i.v.GC treatment period and four after completion of treatment. Three patients had relevant cardiovascular comorbidities. One patient died the day after the last infusion of a treatment course with $1 \mathrm{~g}$ i.v.GC daily for five consecutive days. With the exception of the latter patient, all other patients received a total dose of i.v.GC of at least $8 \mathrm{~g}$ (range 8-15 g).

Severe non-fatal adverse events, mostly a greater than fourfold increase in liver enzymes $(n=20)$ and cardiovascular events $(n=8)$, occurred in 32 patients (Table 2). Relevant comorbidities were reported in half of them. In three of the 20 patients with increased liver enzymes, receiving GC on alternate days every other week, a diagnosis of autoimmune hepatitis was made. All patients stopped i.v.GC and recovered after oral GC therapy. In the majority of cases, the adverse events occurred during GC treatment. The median i.v.GC dose received at the time of the event was $6 \mathrm{~g}$ (range 1-22 $\mathrm{g}$ ).

\section{Discussion}

The purpose of this study was to gather information on the clinical practice of GC treatment for moderateto-severe and active GO and, particularly, to evaluate its side effects. A substantial proportion of the ETA members who linked into the survey without providing a response $(97 / 245,39.6 \%)$ is likely to be those who were not involved in the treatment of GO patients. As a matter of fact, several of the ETA membership includes basic scientists who are not involved in patient care.

The present survey confirms that GC represents, among endocrinologists, the first-line treatment for GO in Europe. Although there is a wide heterogeneity in the treatment modality, the i.v. route is the most common $(7,8)$. This is in keeping with the notion that i.v.GCs are more effective than oral GCs (average response rates $\sim 80$ and $50 \%$ respectively). Indeed, five randomized clinical trials have compared i.v. and oral routes, and all but one showed a greater effectiveness of the former treatment, using a total dose of i.v.GC ranging between 4.2 and $12 \mathrm{~g}(2,3,4,5,6)$. Interestingly, about one-third of physicians treating GO still prefer oral GC.

The survey showed that usual starting dose of i.v.GC was between 0.5 and $1 \mathrm{~g}$, which was maintained for four to six infusions. The most common schedule is based on one weekly infusion, with administration of oral GC in 

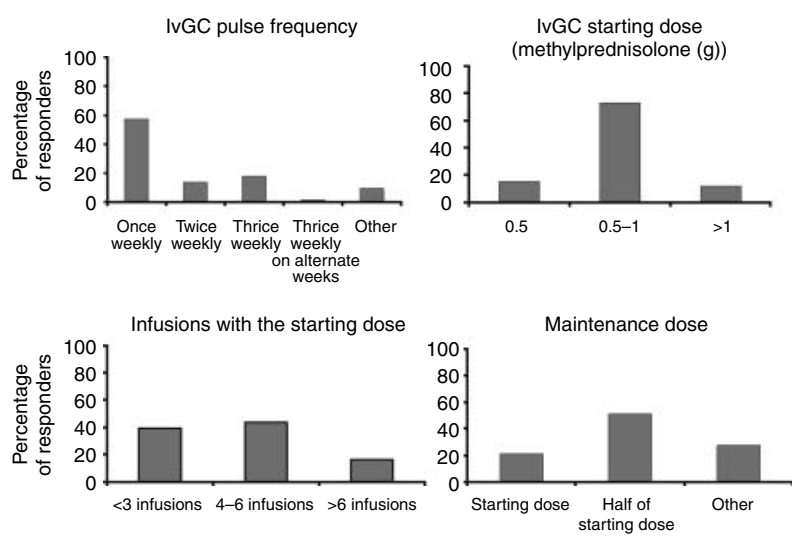

Figure 1 Treatment schedules of i.v.GC therapy used by the 83 European Thyroid Association members.

the interpulse period used by a minority of physicians. The cumulative dose ranged from 0.5 to $12 \mathrm{~g}$ methylprednisolone. The marked heterogeneity of the i.v.GC regimens used by ETA members reflects the present lack of evidence of the superiority of one schedule over others. A wide heterogeneity also exists among oral CG regimens in Europe. The most commonly employed starting dose is between 40 and $75 \mathrm{mg}$, maintained for at least 2 weeks and then gradually tapered down until withdrawal after 3 or more months.

Our study also focused on the side effects of GC therapy. Previous studies comparing oral and i.v.GC have shown that the oral treatment is associated with a significantly higher rate of steroid-related adverse events, particularly weight gain, hypertension, and cushingoid features $(7,8)$. The i.v. route is generally better tolerated, even though some patients complain of palpitations and flushes during or shortly after the infusion (7). The cumulative higher rate of side effects observed using oral GC is supported by this survey, since $81 \%$ of the ETA respondents reported side effects using oral GC and 39\% using i.v.GC. However, severe (nonfatal and fatal) adverse events were more common in i.v.GC, mostly consisting of hepatic and cardiovascular events. Events were fatal in nine cases (seven treated with i.v.GC and two with oral GC). All but one occurred in females, likely reflecting the higher frequency of GO in women compared with men. We cannot estimate the true rate of fatal and non-fatal adverse events in this survey, since data on the total number of patients treated by each ETA member were not available. However, respondents who treated more than 20 patients per year mostly reported fatal events (data not shown). In addition, it seems conceivable that patients receiving i.v.GC are subject to tighter surveillance for side effects than patients receiving oral GC. Indeed, if one considers that, e.g. even serious hepatotoxicity associated with several-fold increases in liver enzymes may be totally asymptomatic, major (nonfatal) adverse events of oral GC may have been underreported or underestimated.

Zang et al. (8) have recently performed a metaanalysis in which the efficacy and morbidity of i.v.GC in GO have been reviewed. Data were largely derived from case-series reports and complete information on side effects was available for 1045 patients. The calculated morbidity and mortality rates were 6.5 and $0.6 \%$ respectively (8). Mild and moderate adverse events mostly accounted for the observed morbidity. The total morbidity rate is rather low when compared with that observed in randomized clinical trials $(43 \%)$. In this regard, it is worth noting that the study that contributed the largest number of patients $(n=800)$ did not mention mild-to-moderate side effects and only focused on acute liver damage (15). Zang et al. reported six cases of death related to i.v.GC and all occurred in patients receiving daily or alternate days injection of a large dose of methylprednisolone ( $1 \mathrm{~g}$ in four cases). Two singlecenter experiences on severe side effects of i.v.GC pulses have been reported. In a study of 101 patients from

Table 2 Characteristics of the 32 reported patients with non-fatal adverse events to treatment with i.v.GC.

\begin{tabular}{|c|c|c|c|c|c|}
\hline \multirow[b]{2}{*}{ Characteristic } & \multicolumn{5}{|c|}{ Non-fatal adverse events } \\
\hline & $\begin{array}{l}\text { Greater than fourfold } \\
\text { increase of liver enzymes }\end{array}$ & Cardiovascular & $\begin{array}{l}\text { Autoimmune } \\
\text { hepatitis }\end{array}$ & $\begin{array}{l}\text { Autoimmune } \\
\text { encephalitis }\end{array}$ & Others \\
\hline$n$ & 19 & 7 & 3 & 1 & 2 \\
\hline Age (years, mean, and range) & $50(21-72)$ & $59(39-76)$ & $41(38-41)$ & 24 & $60(56-64)$ \\
\hline $\begin{array}{l}\text { Mean cumulative dose of i.v.GC } \\
\text { (methylprednisolone }(\mathrm{g}) \text { ) }\end{array}$ & $11.5(2-22)$ & $2.7(1-6)$ & 4.8 & 3.0 & $6.0(4.4-7.6)$ \\
\hline Time of event & & & & NA & \\
\hline During GC & 11 & 7 & 1 & & - \\
\hline After GC & 8 & 0 & 2 & & 2 \\
\hline \multicolumn{6}{|l|}{ Comorbidities } \\
\hline Cardiovascular & 6 & 3 & - & - & - \\
\hline Metabolic & 4 & 1 & 1 & - & 1 \\
\hline Not specified & 3 & 4 & - & - & - \\
\hline None & 9 & 0 & 1 & 1 & 1 \\
\hline
\end{tabular}

NA, Not available. 
Poland, no deaths occurred, but about half of the patients had non-fatal complications, mostly abnormalities of carbohydrate metabolism and infections (16). Among 800 patients from Italy, acute and severe liver hepatoxicity occurred in eight patients $(1 \%)$, three of whom $(0.4 \%)$ died (15). Six of the nine fatal cases described in this questionnaire-based survey, all receiving i.v.GC, had already been reported in the literature (Table 1) $(9,10,11)$. It is worth noting that all seven of the nine patients who died were given either daily or alternate day single doses of $1 \mathrm{~g}$ methylprednisolone. All but one had received a cumulative dose of $8 \mathrm{~g}$ GC or more when the fatal event occurred. The remaining patient received $1 \mathrm{~g}$ methylprednisolone for five consecutive days. Two patients died due to cerebrovascular events during oral GC therapy; in both patients, the total cumulative dose of GC was $2.3 \mathrm{~g}$ (Table 1 ).

Acute liver failure, either during or after completion of therapy, accounted for death in four out of the seven patients receiving i.v.GC $(9,10)$; the remaining three patients died due to cerebrovascular $(n=2)$ or cardiovascular $(n=1)$ events (11). Cardiovascular comorbidities were reported in four out of the five patients who died due to cerebrovascular or cardiovascular events. No history of liver dysfunction was reported in patients who developed fatal liver failure $(9,10)$.

An increase in serum liver enzymes, often asymptomatic, is the most common non-fatal adverse event, which may be related to a direct toxic effect of GC on hepatocytes and appears to be dose dependent $(15,17)$. We cannot exclude that in a minority of cases, an increase in liver enzymes could be due to uncommon liver complications of Graves' disease itself (i.e. transient increase during the thyrotoxic phase or anti-thyroid drug toxicity), even though we clearly requested in the questionnaire to report only adverse events attributed to GC therapy. Autoimmune hepatitis was reported in three patients treated with i.v.GC $(15,18)$. It is likely that a sudden reactivation of the immune system after the abrupt withdrawal of i.v.GC may be responsible for the liver damage. The use of low-dose oral GC therapy either in the interpulse period or after withdrawal of i.v.GC therapy has been advocated to decrease the likelihood of autoimmune hepatitis, particularly in high-risk patients (with positive tests for autoantibodies associated with autoimmune hepatitis) (15), but the evidence that this abolishes the risk of liver damage is unavailable.

High-dose i.v.GC pulse therapy is used in several disorders, including multiple sclerosis and systemic lupus erythematosus; severe cardiovascular adverse events, including death, have been reported in a few cases, mostly related to short infusion rate, ventricular arrhythmias, and/or myocardial infarction $(19,20,21,22)$. An increase in systolic and diastolic blood pressure and in fluid retention might account for the occurrence of severe cardiovascular and cerebrovascular events in patients treated with i.v.GC pulses (12).
A direct evidence-based relationship between highdose oral or i.v.GC and severe fatal or non-fatal side effects remains to be proven. Evidence for the superiority of any of the different i.v.GC schedules is lacking. However, the use of a schedule administering a cumulative dose of $4.5 \mathrm{~g}$ methylprednisolone $(0.5 \mathrm{~g}$ once weekly for 6 weeks, followed by $0.25 \mathrm{~g}$ once weekly for 6 weeks) has gained wide acceptance in recent years, in terms of risk/benefit balance (5). The current recommendation is, however, that the cumulative dose of i.v.GC should not exceed $8 \mathrm{~g}$ in each treatment course and that pulses should not be given on consecutive or alternate days, except in the case of dysthyroid optic neuropathy $(1,8)$. With the exception of dysthyroid optic neuropathy, which requires a very aggressive therapeutic regimen, the administration schedule may also be relevant and a weekly schedule using $<1 \mathrm{~g}$ methylprednisolone per week is recommended $(1,8)$. Patients who are to be treated with high doses of GC should initially be screened for diabetes mellitus and liver dysfunction. To the latter purpose, in addition to liver enzymes, tests for hepatotropic virus markers and autoantibodies related to autoimmune hepatitis are recommended. The potential risk for cardiovascular complications should also be evaluated, by screening the patients for hypokalemia, cardiac arrhythmias, and uncontrolled severe hypertension $(1,8)$. In patients with relevant liver dysfunction (i.e. a greater than fourfold upper normal limit), severe cardiovascular morbidity, severe hypertension, uncontrolled diabetes mellitus and glaucoma, use of i.v.GC therapy should be avoided. When alternative treatments are not indicated, weekly pulses with doses lower than $250 \mathrm{mg}$ appear to be less commonly associated with adverse events, in particular cardiac arrhythmias (20). A slow rate of i.v.GC infusion (60-90 min) should be used and pulse rate and rhythm should be recorded during the infusion. Patients should be carefully followed and monitored for side effects. ECG should be performed before each pulse; tests for liver enzymes should be prescribed every other week during treatment.

This study has several limitations: i) the questionnaire was not formally tested for coverage, applicability, and validity before its use; ii) due to the retrospective nature of the data recollection, the reported adverse events may have occurred over many years, possibly on GC regimens different from those used when the questionnaire was answered; and iii) multiple reporting as some respondents may practice in the same center as other respondents.

In conclusion, i.v.GC appears to be the most common treatment of moderate-to-severe GO among European endocrinologists, and its greater efficacy over oral GC has been proven in randomized clinical trials. As clearly shown also in this survey, this treatment is not devoid of serious side effects, including death. Therefore, in each patient, the potential risks of i.v.GC treatment must be weighed against its potential benefits. Alternative 
therapies should be considered in selected cases, including the use of orbital radiotherapy or the association of low-dose oral GC and cyclosporine (23, 24). Moreover, i.v.GC therapy should only be undertaken in specialized GO centers where a multidisciplinary approach allows the appropriate selection of patients who can benefit from this treatment, as well as facilitating the correct procedure of i.v.GC administration and monitoring of possible adverse events. A strict follow-up schedule should be applied to patients receiving high-dose oral or i.v.GC for the treatment of $\mathrm{GO}$, in view of the often asymptomatic features of acute liver damage.

\section{Declaration of interest}

The authors declare that there is no conflict of interest that could be perceived as prejudicing the impartiality of the research reported.

\section{Funding}

This research did not receive any specific grant from any funding agency in the public, commercial or not-for-profit sector.

\section{Acknowledgements}

We thank all members of the European Thyroid Association who answered the questionnaire and made this study possible. We particularly thank the following members for providing additional information on the patients they reported with severe adverse events: Biljana Beleslin (Serbia), Mustafa Cesur (Turkey), Murat Erdogan (Turkey), Gianfranco Fenzi (Italy), Fadila Gadalla (Egypt), Laslo Hegedus (Denmark), Alicia Hubalewska-Dydejczyk (Poland), Venetsana Kyriazopoulou (Greece), Bojan Lozanov (Bulgaria), Birte Nygaard (Denmark), Maryia Petrova (Bulgaria), Patrice Rodien (France), Leif Tallstedt (Sweden), Bozo Trbojevc (Serbia), Guia Vannucchi (Italy), Michael Weissel (Austria), and Silvia Wengrowicz (Spain). We also thank all EUGOGO members for contributing to the formulation of the questionnaire or helpful discussion during the preparation of the manuscript.

\section{References}

1 Bartalena L, Baldeschi L, Dickinson AJ, Eckstein A, KendallTaylor P, Marcocci C, Mourits MP, Perros P, Boboridis K, Boschi A, Currò N, Daumerie C, Kahaly GJ, Krassas G, Lane CM, Lazarus JH, Marinò M, Nardi M, Neoh C, Orgiazzi J, Pearce S, Pinchera A, Pitz S, Salvi M, Sivelli P, Stahl M, von Arx G \& Wiersinga WM. Consensus statement of the European group on Graves' orbitopathy (EUGOGO) on management of Graves' orbitopathy. European Journal of Endocrinology 2008158 273-285. (doi:10.1530/EJE07-0666)

2 Marcocci C, Bartalena L, Tanda ML, Manetti L, Dell'Unto E, Rocchi R, Barbesino G, Mazzi B, Bartolomei MP, Lepri P, Cartei F, Nardi M \& Pinchera A. Comparison of the effectiveness and tolerability of intravenous or oral glucocorticoids associated with orbital radiotherapy in the management of severe Graves' ophthalmopathy: results of a prospective, single-blind, randomized study. Journal of Clinical Endocrinology and Metabolism 200186 3562-3567. (doi:10.1210/jc.86.8.3562)

3 Macchia PE, Bagattini M, Lupoli G, Vitale M, Vitale G \& Fenzi G. High-dose intravenous corticosteroid therapy for Graves' ophthalmopathy. Journal of Endocrinological Investigation 2001 24 152-158.
4 Kauppinen-Makelin R, Karma A, Leinonen E, Loyttyniemi E, Salonen O, Sane T, Setala K, Viikari J, Heufelder A \& Valimaki M. High dose intravenous methylprednisolone pulse therapy versus oral prednisone for thyroid-associated ophthalmopathy. Acta Ophthalmologica Scandinavica 200280 316-321. (doi:10.1034/j. 1600-0420.2002.800316.x)

5 Kahaly GJ, Pitz S, Hommel G \& Dittmar M. Randomized, single blind trial of intravenous versus oral glucocorticoid monotherapy in Graves' orbitopathy. Journal of Clinical Endocrinology and Metabolism 200590 5234-5240. (doi:10.1210/jc.2005-0148)

6 Aktaran S, Akarsu E, Erbagci I, Araz M, Okumus S \& Kartal M. Comparison of intravenous methylprednisolone therapy vs. oral methylprednisolone therapy in patients with Graves' ophthalmopathy. International Journal of Clinical Practice 200761 45-51. (doi:10.1111/j.1742-1241.2006.01004.x)

7 Stiebel-Kalish H, Robenshtok E, Hasanreisoglu M, Ezrachi D, Shimon I \& Leibovici L. Treatment modalities for Graves' ophthalmopathy: systematic review and metaanalysis. Journal of Clinical Endocrinology and Metabolism 200994 2708-2716. (doi:10.1210/jc.2009-0376)

8 Zang S, Ponto KA \& Kahaly GJ. Clinical review: intravenous glucocorticoids for Graves' orbitopathy: efficacy and morbidity. Journal of Clinical Endocrinology and Metabolism 201196 320-332. (doi:10.1210/jc.2010-1962)

9 Weissel M \& Hauff W. Fatal liver failure after high-dose glucocorticoid pulse therapy in a patient with severe thyroid eye disease. Thyroid 200010521.

10 Marinó M, Morabito E, Brunetto MR, Bartalena L, Pinchera A \& Marocci C. Acute and severe liver damage associated with intravenous glucocorticoid pulse therapy in patients with Graves' ophthalmopathy. Thyroid 200414 403-406. (doi:10.1089/ 105072504774193276)

11 Lendorf ME, Rasmussen AK, Fledelius HC \& Feldt-Rasmussen U. Cardiovascular and cerebrovascular events in temporal relationship to intravenous glucocorticoid pulse therapy in patients with severe endocrine ophthalmopathy. Thyroid 200919 1431-1432. (doi:10.1089/thy.2009.0069)

12 Gursoy A, Cesur M, Erdogan MF, Corapcioglu D \& Kamel N. New-onset acute heart failure after intravenous glucocorticoid pulse therapy in a patient with Graves' ophthalmopathy. Endocrine 200629 513-516. (doi:10.1385/ENDO:29:3:513)

13 Owecki M \& Sowiński J. Acute myocardial infarction during highdose methylprednisolone therapy for Graves' ophthalmopathy. Pharmacy World Science 200628 73-75. (doi:10.1007/s11096006-9013-y)

14 SAS Institute, Inc. SAS/STAT ${ }^{\circledR} 9.1$ User's Guide. Cary, NC: SAS Institute, Inc., 2004.

15 Marinò M, Morabito E, Altea MA, Ambrogini E, Oliveri F, Brunetto MR, Pollina LE, Campani D, Vitti P, Bartalena L, Pincheral A \& Marcocci C. Autoimmune hepatitis during intravenous glucocorticoid pulse therapy for Graves' ophthalmopathy treated successfully with glucocorticoids themselves. Journal of Endocrinological Investigation 200528 280-284.

16 Kulig G, Kaźmierczyk-Puchalska A, Krzyzanowska-Swiniarska B \& Pilarska K. Complications during systemic corticosteroid therapy combined with orbital radiotherapy in patients with Graves orbitopathy. Przeględ Lekarski 200461 855-856.

17 Le Moli R, Baldeschi L, Saeed P, Regensburg N, Moutits MP \& Wiersinga WM. Determinants of liver damage associated with intravenous methylprednisolone pulse therapy in Graves' ophthalmopathy. Thyroid 200717 357-362. (doi:10.1089/thy.2006. 0267)

18 Salvi M, Vannucchi G, Sbrozzi F, Del Castello AB, Carnevali A, Fargion S \& Beck-Peccoz P. Onset of autoimmune hepatitis during intravenous steroid therapy for thyroid-associated ophthalmopathy in a patient with Hashimoto's thyroiditis: case report. Thyroid 200414 631-634. (doi:10.1089/1050725041692927)

19 Guillen EL, Martinez Ruiz A \& Vidal Bugallo JB. Hypotension, bradycardia, and asystole after high-dose intravenous 
methylprednisolone in a monitored patient. American Journal of Kidney Diseases 199832 E4. (doi:10.1053/ajkd.1998.v32.pm 10074612)

20 Erstad BL. Severe cardiovascular adverse effects in association with acute, high-dose corticosteroid administration. DICP: the Annals of Pharmacotherapy 198923 1019-1023.

21 Wollheim FA. Acute and long-term complications of corticosteroid pulse therapy. Scandinavian Journal of Rheumatology $1984 \mathbf{5 4}$ 27-32. (doi:10.3109/03009748409103858)

22 Bocanegra TS, Castañeda MO, Espinoza LR, Vasey FB \& Germain BF. Sudden death after methylprednisolone pulse therapy. Annals of Internal Medicine 198195122.
23 Bartalena L \& Tanda ML. Clinical practice. Graves' ophthalmopathy. New England Journal of Medicine $2009360994-1001$. (doi:10.1056/NEJMcp0806317)

24 Bartalena L. What to do for moderate-to-severe and active Graves' orbitopathy if glucocorticoids fail? Clinical Endocrinology 201073 149-152. (doi:10.1111/j.1365-2265.2010.03783.x)

Received 2 September 2011

Revised version received 24 October 2011

Accepted 4 November 2011 